\title{
A Period Analysis of the Optical Line Variability of $\beta$ Cephei ${ }^{1}$
}

\section{J. Telting}

Astronomical Institute 'Anton Pannekoek', University of Amsterdam, Kruislaan 403, NL-1098 SJ Amsterdam, Netherlands

C. Aerts ${ }^{2}$, and P. Mathias ${ }^{3}$

Instituut voor Sterrenkunde, Katholieke Universiteit Leuven, Celestijnenlaan 200 B, B-3001 Heverlee, Belgium

\section{Introduction}

Recently Aerts et al. (1994, Paper I) discovered multi-periodicity in the variations of optical lines of $\beta$ Cephei. They identified the frequency of the radial pulsation $\left(f_{1}=5.25 \mathrm{cycles} /\right.$ day $)$, and additionally found 2 frequencies $\left(f_{2}=\right.$ $5.38 \mathrm{c} / \mathrm{d}, f_{3}=4.92 \mathrm{c} / \mathrm{d}$ ) close to the main frequency. The amplitude of these variations was found to be much smaller than that of the radial pulsation. Aerts et al. (1994) attributed these small variations at the newly found frequencies to non-radial pulsation modes of the star, but labelled the mode-identifications as uncertain.

As a follow up on this work, we investigated the whole frequency range that can be analysed with the data set described in Paper I. For four absorption lines (Si III $\lambda 4552,4567,4574$ and $O$ II $\lambda$ 4591) we examined variations in EW and the velocity moments of the absorption lines (yielding the velocity shift, line width and skewness). We also investigated the variations of the normalised intensity for each individual wavelength bin of the spectra.

\section{Results}

We find, apart from the already known frequencies, two other frequencies $\left(f_{4}=\right.$ $5.08 \mathrm{c} / \mathrm{d}, f_{5}=5.42 \mathrm{c} / \mathrm{d}$ ) around the main frequency, which have variations exceeding the noise level. These frequencies clearly show up in the CLEANed periodograms of the first velocity moment and of the intensity variations across the lines. Furthermore, we find a peak in the periodograms of the moments at $0.17 \mathrm{c} / \mathrm{d}$, corresponding to a period of 6 days.

Henrichs et al. (1993) and Veen et al. (1995) identify the frequency of variations detected in UV wind lines with the rotation period of a magnetic dipole,

\footnotetext{
${ }^{1}$ Based on observations obtained at the Observatoire de Haute-Provence, France

${ }^{2}$ Senior Research Assistant, Belgian National Fund for Scientific Research

${ }^{3}$ Present Address: Observatoire de la Côte d'Azur, Département Fresnel, BP 229, F-06304 Nice Cedex 4, France
} 
which implies a rotation period of the star of 12 days. We find that the spacings between the main frequency $f_{1}$ and the frequencies $f_{3}, f_{4}$, and $f_{5}$ are multiples of the frequency detected in the UV wind lines. This means that we can identify the spacing between the frequencies (i.e., $f_{1}-f_{3} \approx 0.33 \mathrm{c} / \mathrm{d}, f_{1}-f_{4} \approx$ $0.17 \mathrm{c} / \mathrm{d}, f_{5}-f_{1} \approx 0.17 \mathrm{c} / \mathrm{d}$ ), as derived from the optical Sill and OII lines, as multiples of the rotation period.

We find that for the intensity variations across the lines, the pattern of frequencies $\left(f_{2}, f_{3}, f_{4}\right.$ and $\left.f_{5}\right)$ around the frequency $f_{1}$ of the main (radial) pulsation, repeats itself around every detected harmonic of the main frequency, with the same frequency spacing as around the main frequency.

\section{Discussion}

The pattern of frequency combinations of $f_{1}$ and $f_{2}$ (i.e., $f_{1}, f_{2}, f_{1}+f_{1}, f_{1}+f_{2}$, $2 f_{1}+f_{1}, 2 f_{1}+f_{2}$, etc.), as detected in the intensity variations across the lines, can be modelled as a multi-periodic star with $\mathrm{l}=\mathrm{m}=0$ and $\mathrm{l}=\mathrm{m}=2$ spheroidal modes (Paper I). This model also gives a good description of the observed power and phase distribution across the absorption lines. At present we are investigating if the power and phase distribution of the frequencies $f_{3}, f_{4}$ and $f_{5}$ can be modelled as the result of (non-)radial pulsations.

The fact that the spacings of the detected frequencies are multiples of the rotation frequency suggests that the observed variations with frequencies $f_{3}$, $f_{4}$, and $f_{5}$ might be due to modulation effects. The similarities between the variations of UV wind lines and optical lines suggest that either a common phenomenon in wind and photosphere (e.g., a magnetic field) causes these similarities or that one type of variation (wind variability) may be caused by the other (pulsations).

Further investigations will hopefully show whether the variations with frequencies $f_{3}, f_{4}$, and $f_{5}$ in the spectra of $\beta$ Cephei are best described as (non)radial pulsations, or as the result of modulation caused by the combination of rotation, pulsations and the presence of a magnetic field.

\section{References}

Aerts, C., Mathias, P., Gillet, D., \& Waelkens, C., 1994, A\&A 286, 109

Henrichs, H.F., Bauer, F., Hill, G.M., Kaper, L., Nichols Bohlin, J.S., \& Veen P.M., 1993, Proceedings IAU Coll. 139, p. 186, Cambridge Univ. Press

Veen, P.M., Henrichs, H.F., et al. 1995, in preparation 